Voix et Images

volxetimages

\title{
La Postérité littéraire du Plan de développement de l'Est du Québec
}

\section{Gilles Lamontagne}

Volume 3, numéro 3, avril 1978

Pierre Perrault

URI : https://id.erudit.org/iderudit/200125ar

DOI : https://doi.org/10.7202/200125ar

Aller au sommaire du numéro

Éditeur(s)

Les Presses de l'Université du Québec

ISSN

0318-9201 (imprimé)

1705-933X (numérique)

Découvrir la revue

Citer cet article

Lamontagne, G. (1978). La Postérité littéraire du Plan de développement de

l'Est du Québec. Voix et Images, 3(3), 467-480. https://doi.org/10.7202/200125ar d'utilisation que vous pouvez consulter en ligne.

https://apropos.erudit.org/fr/usagers/politique-dutilisation/ 


\section{La Postérité littéraire du}

\section{Plan de développement de l'Est du Québec}

La Gaspésie, c'est un bel endroit pour naître et mourir. - Et entre les deux?

- Entre les deux, il faut quitter...

Charles Soucy, À travers la mer, p. 25.

Lorsque le ministère de l'Agriculture de la Province mit sur pied le Bureau d'aménagement de l'Est du Québec (BAEQ), en 1963, il ne se doutait pas qu'une dizaine d'années plus tard, il aurait déjà lui-même ainsi que les planificateurs du BAEQ et leur créature, le Plan, suscité l'apparition d'un embryon de mythologie littéraire. Trois romans écrits et publiés au début de la décennie en cours par des auteurs originaires du Bas-SaintLaurent et de la Gaspésie présentent en effet des efforts convergents d'imagination simplificatrice allant dans ce sens.

Le premier de ces romans, la Marche des grands cocus de Roger Fournier', exploite l'échec des politiques gouvernementales en matière d'agriculture. Le deuxième, C't'à cause qu'y vont su'a lune de Gilbert Langlois ${ }^{2}$, apparaît comme une démythification du tourisme considéré comme la panacée contre tous les maux économiques dont sont affligés le BasSaint-Laurent et la Gaspésie. Enfin, le roman de Charles Soucy, À travers la mer $^{3}$, illustre à sa façon la détérioration accélérée de l'industrie de la pêche dans l'Est du Québec.

Ce qui est proposé ici, c'est une lecture partielle - donc partiale de ces trois romans afin de mettre en évidence quelques éléments d'une matière littéraire peut-être appelée à proliférer pour se transformer en une sorte de saga du Plan.

Pour le bénéfice du lecteur, il ne sera sans doute pas inopportun de rappeler brièvement que le BAEQ élabora, entre 1963 et 1966, un volumineux rapport, le Plan, en dix cahiers totalisant plus de 2000 pages et incluant 231 recommandations relatives au développement des principaux secteurs d'activité de l'Est du Québec, c'est-à-dire des neuf circonscriptions électorales provinciales du Bas-Saint-Laurent, de la Gaspésie et des îles de.la Madeleine. La publication de ce rapport fut précédée d'une Esquisse du Plan plus imposante encore puisqu'elle comprenait 2500 pages de texte auxquelles venait s'ajouter une quarantaine d'annexes tech- 
niques. Le tout au coût approximatif de quatre millions de dollars. Terminé en 1966, le Plan, destiné à atténuer sinon à faire disparaître l'écart entre le niveau économique de l'Est du Québec et celui de la Province, ne connaîtra un début de mise en application que deux ans plus tard, soit en mai 1968. A cette date, en effet, et dans un premier temps, les gouvernements provincial et fédéral s'engageaient à injecter conjointement la somme de $\$ 411318000$ dans ce qu'il fut désormais convenu d'appeler le «Territoire pilote d'aménagement ${ }^{4} »$.

Si maintenant nous jetons un coup d'œil sur la situation qui a prévalu dans trois secteurs clés étudiés par les planificateurs - l'agriculture, le tourisme et la pêche - au cours des premières années qui ont suivi la mise en route du Plan, nous pourrons nous faire une idée de la réalité concrète transformée par l'imaginaire de nos trois romans.

Rappelons d'abord que le BAEQ avait préconisé une revalorisation de l'agriculture fondée sur la réalisation de quatre programmes précis dont l'un visait plus spécifiquement la normalisation de la structure des fermes. Ce programme, confié plus tard à la responsabilité d'un Service du réaménagement foncier suscita beaucoup d'espoir chez les agriculteurs; tant et si bien que sous l'impulsion de ce service

des comités locaux de réaménagement s'étaient formés dans une dizaine de paroisses. Les cultivàteurs et représentants d'organismes socio-économiques qui en faisaient partie devaient, avec l'aide technique du Ministère, bâtir des plans d'aménagement pour leur municipalité. Mais l'impuissance du Service à donner suite aux projets ébauchés a vite fait cesser les activités ${ }^{5}$.

Bien plus: en axant le programme presque exclusivement sur l'achat des fermes marginales, le Service de réaménagement foncier se trouva à contribuer davantage à la fermeture de localités qu'au développement de l'agriculture. De sorte que le bilan des politiques agricoles, en 1971, était en gros le suivant: 10 localités fermées; le nombre des fermes passé de 13278 qu'il était en 1961 à 7066 dont 1868 seulement effectuaient des ventes de $\$ 5000$ et plus par année. On était donc loin de l'objectif fixé par le BAEQ pour 1977: 5785 agriculteurs à plein temps, c'est-à-dire retirant de leurs fermes la totalité de leurs revenus de subsistance!

Entre autres ombres au tableau de la situation agricole sur le territoire pilote: l'entêtement des agriculteurs à continuer de miser sur la production laitière qui, en 1970 , représentait $68 \%$ du total de la production agricole régionale. La politique de contingentement pratiquée par. le gouvernement fédéral ne semble pas être parvenue à modifier la situation en faveur de la production bovine, du moins pas encore en 1971 puisque cette dernière ne représentait alors que $5,7 \%$ du total de la production agricole.

Cette situation d'ensemble est essentiellement celle dans laquelle se débat le fonctionnaire Sainclair au début du roman de Roger Fournier, la Marche des grands cocus. 
Sainclair parlait [...] des projets de développement qui avaient été mis en marche par les divers gouvernements, des millions qui avaient été mis à la disposition du Bas-du-Fleuve par deux Premiers ministres, du problème du lait, qui était capital, du reboisement [...], du démantèlement de certaines paroisses qu'il fallait absolument fermer (p. 37).

Complètement débordé, il appelle Québec au secours. On lui envoie Lucien Lambert, un fonctionnaire supérieur soi-disant efficace. En arrivant à Rimouski, ce dernier se rend au bureau de Sainclair qui lui esquisse un rapport verbal des plus déprimants. Fortement ébranlé par ce qu'il vient d'entendre, Lucien songe un instant à retourner tout de suite à Québec ouvrir les yeux de ses supérieurs s'il en est encore temps. Mais il se ravise et demande plutôt à Sainclair de l'amener rencontrer les agriculteurs de la région afin de pouvoir se faire une idée précise des problèmes qui les confrontent. En compagnie de son collègue, il visite donc rapidement quelques petites paroisses s'arrêtant ça et là pour causer un peu avec les gens. Au fur et à mesure qu'il s'enfonce dans les terres, la réalité qu'il découvre lui donne l'impression de se trouver "dans un' autre pays" ( $p$. 41 ), sur « un autre continent» (p. 46), dans “un autre monde» (p. 47). Au $4^{\circ}$ rang de Saint-Antime, c'est le choc: Philippe Côté, un cultivateur particulièrement réfractaire aux politiques du Plan, vient de se suicider; on l'a trouvé pendu dans sa grange au petit cable de sa fourche à foin. Accouru sur place, Josué, le leader naturel de la paroisse, n'hésite pas à dégager la cause du drame:

Si Philippe s'est pendu [...], c'est parce qu'y a rien qui marche comme y faut dans not' maudit pays [...] Sa terre le faisait pas vivre, pis si sa terre le faisait pas vivre, c'est parce que c'est mal organisé dans not' c... de province (p. 48).

Au moment des funérailles de Philippe, Josué est encore plus explicite:

Y a été tué par tout le monde qui fait rien, par ceux qui font semblant de nous diriger. Sainclair, c'est un bon garçon, et aujourd'hui $y$ 'est icitte avec un gars de Québec. Ce monde-là, ça vient nous voir de temps en temps pour parler, pour prendre des notes dans des p'tits carnets, ça repart pis on n'entend pus parler de rien. Faut que ça finisse ces singeries-là. O.K. Sainclair? (p. 69).

Le suicide de Philippe précipite une série de réactions en chaîne qui constitue la trame du roman. D'abord, la révolte de Josué. Au cours des funérailles, il abat tout son troupeau de vaches sur la place de l'église ${ }^{6}$. Puis, on assiste à la radicalisation progressive de Lucien et de Sainclair qui se rangent du côté des agriculteurs en colère. Au cours d'un grand rassemblement populaire convoqué par Sainclair à Rimouski pour tâcher de trouver des solutions aux problèmes des gens, Isabelle, la maîtresse de Lucien, propose une marche sur Québec ${ }^{7}$. Aussitôt décidée, la marche s'organise. Transformée en pasionaria, Isabelle anime et dirige plus de 8000 (!) marcheurs. A Québec, au moment où le Premier ministre s'apprête à rencontrer les contestataires, Lucien tire à bout portant sur le ministre de l'Agriculture et le tue. Riposte immédiate de la police qui abat Lucien. Appelée en renfort, l'armée disperse les marcheurs non sans avoir fait un bon nom- 
bre d'autres victimes, dont Sainclair. Isabelle s'étant réfugiée dans son appartement, à Québec justement, Josué, avec lequel elle s'est liée au cours de la grande marche, vient l'y rejoindre. Ils passent la nuit ensemble et au petit matin, Isabelle supplie Josué de. la tuer avec sa carabine. Josué accepte après quoi il s'enfuit prendre le train à Lévis pour rentrer à Rimouski.

Si la Marche des grands cocus se présente comme une transposition romanesque de l'échec des politiques ministérielles, en matière de réaménagement agricole, l'humour presque noir du roman de Gilbert Langlois, C't'à cause qu'y vont su'a lune n'est pas sans rappeler la naïveté sinon l'euphorie délirante de certains planificateurs en matière de tourisme.

L'immense potentiel touristique de la Gaspésie est réel, mais il ne semble pas avoir fait l'objet de politiques très imaginatives ni très cohérentes de la part des organismes gouvernementaux. Le Plan avait bien recommandé l'aménagement de trois centres principaux devant constituer autant d'étapes pour les touristes qui font le tour de la Péninsule. Mais ici encore, toutes sortes de lenteurs et de complications administratives sont venues plus ou moins compromettre le développement de ce secteur. A cet égard, le projet du parc de Bic est typique:

Cinq projets d'aménagement ont été réalisés depuis 1963. Ne voulant pas encore passer à l'action au début de 1973, l'ODEQ (Office de développement de l'Est du Québec) a engagé $\$ 100000$ pour de nouvelles études. Lorsque ces dernières ont été terminées, on a décidé de prévoir une autre somme importante d'argent pour faire effectuer de nouvelles études. C'est par dizaines que les rapports de recherche du secteur touristique s'accumulent sur les tablettes. Le budget de l'Entente s'épuise rapidement et il y a très peu de réalisations. Parmi celles effectuées, la plupart n'ont aucun effet de rétention du tourisme et certaines ne visent qu'à satisfaire des besoins en loisirs purement locaux ${ }^{8}$.

En 1971, le rapport Métra, confirmé en cela par le ministère de l'Industrie et du Commerce, estimait que par comparaison avec 1964, la région enregistrait une diminution des équipements d'accueil pour les touristes. Bien sûr, le parc Forillon a depuis été créé. Mais une fois de plus, il semble bien que certains planificateurs se soient montrés trop enthousiastes allant même jusqu'à prétendre sans broncher que sa réalisation créerait 1000 emplois permanents! Les statistiques des autorités du parc sont cependant d'un tout autre ordre: elles indiquent qu'il procurait en fait 15 emplois permanents et 150 temporaires et cela, durant la phase d'exécution des travaux ${ }^{9}$ !

Pratiquement, l'industrie touristique sur le territoire pilote se confond largement avec le camping puisque la moitié environ des voyageurs qui circulent dans la région s'y livrent. Or il est démontré que cette forme de tourisme procure des revenus faibles dont les retombées économiques, notamment en termes de salaires, s'avèrent négligeables. Pourtant, on continue volontiers d'entretenir une sorte de mythe de la grande rentabilité 
du camping, mythe parfois cruellement contredit par la réalité. Par exemple, on a construit à grands frais une jetée en face de Carleton pour y aménager un terrain destiné aux campeurs. Des statistiques de 1972 révèlent un taux d'occupation de $27,5 \%$ pour une durée moyenne de 1,23 jour!

Anthyme (Thyme), le personnage principal de C't'à cause qu'y vont su'a lune est à sa façon une victime de ce mythe. II exploite à proximité de Sainte-Anne-des-Monts son propre terrain de camping dont les revenus lui permettent tout juste de vivoter, lui et sa femme Henrietta. L'annonce d'une éclipse totale du soleil dans cette région se présente comme une occasion inespérée d'améliorer enfin son sort en tirant profit de la foule d'observateurs et de curieux que ne manquera pas d'attirer pareil événement. Afin de pouvoir répondre aux besoins de tout ce monde, Thyme va même jusqu'à décider de faire construire une épicerie sur son terrain. Tel que prévu, le jour venu les gens affluent de partout ( $y$ compris de Cap Kennedy!) et Thyme peut pour la première fois de sa vie afficher complet. Malheureusement, un nuage vient masquer l'éclipse pendant les 160 précieuses secondes de sa durée, précipitant du même coup la désertion des campeurs. Doublement déçu d'avoir raté un beau spectacle et surtout une affaire qui promettait d'être bien meilleure (il avait espéré qu'attirés par l'éclipse, les touristes en profiteraient pour passer au moins une partie de leurs vacances chez lui), Thyme va se coucher en bougonnant: "Si ça va mal de même [...] si i a pas eu d'éclisse, [...] c't'à cause qu'y vont su'a lune!" (p. 163).

Ainsi, pas plus dans ce roman que dans la réalité, le tourisme et plus particulièrement le camping ne sont le pactole. Reste la pêche.

Si on a pu affirmer, au moment où le BAEQ amorçait ses travaux en 1963 que "l'association traditionnelle que l'on faisait entre la Gaspésie et la mer n'avait déjà plus de sens ${ }^{10} \mathrm{~m}$, doit-on supposer qu'elle en avait encore moins en 1974, année de la publication du roman de Charles Soucy, À travers la mer? Pourtant, la pêche a toujours été et demeure encore à peu près le seul secteur de l'économie de la région pilote à ne pas subir la concurrence des grandes agglomérations urbaines. On s'attendrait donc à ce qu'elle constitue le principal pilier de l'économie gaspésienne. Qu'en est-il au juste? Malheureusement, il est difficile de le savoir"1. Chose certaine, le secteur s'est profondément modifié, mais dans quelle mesure s'est-il industrialisé ? Voyons plutôt. En 1964, la Gaspésie comptait 1335 pêcheurs dont 400 hauturiers. Dix ans plus tard (1974), ce nombre était passé à 2330 dont 1621 hauturiers. Or le Plan avait prévu qu'en 1971, les pêcheurs de la Péninsule ne seraient plus que 835 au total par suite de l'application des politiques de regroupement et d'industrialisation. Dans la mesure où, en se fondant sur les chiffres seulement "on peut parler de progrès, un tel progrès serait davantage attribuable à l'initiative des pêcheurs èx-mêmes et des compágnies de transformation du pois- 
son qu'aux interventions gouvernementales. A titre d'exemple de nouvelles lenteurs administratives, on note que

la rationalisation des points de débarquement se fait toujours attendre de même que l'organisation des parcs industriels de pêche promis depuis 1970 à Rivière-au-Renard, Grande-Rivière, Paspébiac et Capaux-Meules ${ }^{12}$.

Quoi qu'il en soit, les transformations dont fut l'objet le secteur des pêches en Gaspésie ne se sont pas opérées sans contrecoups surtout, semble-t-il, pour les pêcheurs côtiers. En faisant le tour de la Péninsule, le voyageur peut facilement conclure en ce sens lorsqu'il aperçoit des dizaines de petits quais partiellement emportés par les vagues.

Benoît Lelièvre, le personnage central de À travers la mer est l'un de ces pêcheurs qui sont frustrés. II vit à Pabok (Pabos?), quelque part entre Newport et Grande-Rivière. Fils de pêcheur, il est profondément attaché à son métier et à la mer dont il parle volontiers en poète. II souffre de voir la pêche, du moins celle de type traditionnel, dépérir: «ll y avait eu une époque où l'anse était couverte de barques. Aujourd'hui, il n'y en avait presque plus» (p. 13). Effectivement, plusieurs maisons abandonnées dans le village témoignent de nombreux départs, le dernier étant celui du jeune Alain, compagnon de pêche de Benoît. La femme de celuî-ci, Anna, apparaît au début du roman comme un être solitaire, silencieux. Elle ne prend plus beaucoup de plaisir aux sorties hebdomadaires à Percé dans un bar le samedi soir. Bref, elle se dit fatiguée de «ce maudit pays» au point d'en dépérir physiquement. Tant et si bien qu'elle finit par convaincre son mari de tout vendre et de partir à Montréal. Alors qu'au contact de la ville Anna renaît, Benoît réagit de plus en plus mal à son déracinement. Dès son arrivée, les périodes de chômage vont alterner avec des emplois épisodiques qu'il n'aime pas: messager, aide dans un supermarché; déneigeur... De plus en plus malheureux, il se met à boire. Entretemps, Anna s'est trouvé un emploi dans une manufacture. Puis le silence, un silence de plus en plus lourd, ponctué de reproches, voire d'explosions de haine, s'installe progressivement entre Benoît et sa femme. Finalement, ils en viennent à reconnaître qu'ils ne s'aiment plus. Après avoir cherché un exutoire du côté de sa mémoire volontaire en s'efforçant de “réveiller tout un passé pour passer le temps» (p. 90), Benoît se révèle de plus en plus obsédé par la hantise de son village natal, de la mer, de la pêche, de son compagnon James resté là-bas et à qui il a abandonné sa barge. Finalement, avant qu'un jour Anna en rentrant de son travail ne retrouve Benoît allongé mort sur la tapis du salon, le roman emprunte le rythme d'une séquence de film au ralenti; l'idée même que James ait pu lui aussi quitter Pabok fait chavirer Benoît dans le cauchemar:

II tomba sur le dos et sa barge vint bomber sur lui. Sa dernière pensée fut: le sable ne sera jamais assez élastique pour me protéger. La mer monta et vint lécher sa joue ( $p$. 144).

Voilà donc comment les sujets de nos romans considérés individuellement se présentent par rapport aux préoccupations majeures du Plan. 
II sera maintenant intéressant de chercher à déceler quelques-unes des caractéristiques de l'univers que composent les trois romans superposés.

D'abord, on constate d'un roman à l'autre des recoupements et une complémentarité dans l'évocation du cadre spatial qui vont dans le sens suivant: la mer, la forêt, les montagnes, les champs incarnent la beauté même objectivement, c'est-à-dire du point de vue de l'étranger, du touriste. II arrive parfois à ceux qui vivent dans un tel paysage de prendre conscience de sa splendeur; il peut même leur arriver d'en retirer un plaisir presque physique qui se confond avec un sens très profond de l'appartenance. C'est le cas de Benoît dans À travers la mer. Tout en partageant ses occupations entre la culture de son lopin de terre, la pêche et les travaux en forêt l'automne, il considère la mer et la terre et les travaux en forêt l'automne, il considère la mer et la terre comme des maîtresses, sa préférée étant la mer. Benoît est donc de ceux qui appartiennent à leur coin de pays dans le sens où les "Acadiens, quand ils veulent savoir d'où vient quelqu'un [demandent]: à quelle région appartiens-tu" (p. 96). On comprend alors le sentiment de culpabilité de Benoît une fois rendu à Montréal; il a l'impression d'avoir trahi quelqu'un, d'avoir vendu son âme en quittant sa barge et sa terre. Dans les trois romans, l'attitude de Benoît est toutefois exceptionnelle tout comme, d'ailleurs, celle de sa femme qui, à l'autre extrême, suffoque littéralement dans le paysage gaspésien. Pour elle, la Gaspésie est a un pays impossible [...] dont la situation géographique [n'est] pas un début, mais une fin" (p. 59): la mer y “détruit l'homme par son manque d'appui » (p. 57)". - Isabelle, dans la Marche des grands cocus, a la même attitude: "Le fleuve est trop grand, dit-elle, il nous détruit" (p. 58). - Surtout, la femme de Benoît, comme Thyme, en veut à l'hiver à cause de sa rigueur et de sa longueur bien sûr, mais surtout à cause du silence insupportable qu'il installe pour six mois sur la Péninsule. L'arrivée de cette saison lui donne envie de ne plus se lever.

Parfois, le brouillard transforme la beauté du décor en un paysage inquiétant, voire menaçant. Par exemple, lorsque Lucien approche de Bic en route vers Rimouski, il voit dans les montagnes déchiquetées par la brume un spectacle prémonitoire. Effectivement, le lendemain lorsqu'il cheminera de Pointe-au-Père à Saint-Antime avec Sinclair, il aura l'impression de découvrir un paysage hostile, une terre de Caïn, un pays sans horizon avec "des savanes autour et, en avant, des montagnes, des côtes, des bosquets, des «tas de roches" au milieu des champs» (p. 42). Au $4^{e}$ rang de Saint-Antime, quelques fonds de terre noire, une dizaine de fermes dont la moitié ont l'air plus ou 'moins abandonnées. C'est ici que Philippe s'est pendu. .

D'une manière générale cependant, l'attitude qui caractérise la plupart des personnages par rapport au cadre géographique dans lequel ils évoluent consiste en un mélange d'indifférence et d'hébétude. Cette situation est sans doute due au fait que le paysage subit une sorte de défiguration, de dégradation morale imputable à la réalité socio-économique qui prévaut dans la région. 
On se rappellera comment Benoît, à Pabok, parvenait tout juste à "survivre" (le mot est de sa femme) en partageant ses activités entre la culture de la terre, la pêche et le travail en forêt. Son cas est loin d'être exceptionnel si l'on en juge entre autres par cette bribe de conversation entre Lucien et Sainclair traversant le village de Pointe-au-Père:

- De quoi vivent les gens d'ici?

- Ils ne vivent pas... Ils vivotent... Un tout p'tit peu de culture, mais surtout, l'assistance sociale...

- Mais! Ils doivent bien pêcher, non?

- Les enfants, à partir du mois d'août, viennent pêcher l'éperlan, ici sur le quai... Mais la pêche, non, c'est pas organisé (p. 41).

Avant son mariage avec Henrietta, Thyme devait lui aussi s'exiler périodiquement pour aller travailler dans les forêts de la Côte Nord. A cinquante ans, il se retrouve avec «in camping pis queque cennes de côté " (VL, p. 74).

Dans l'arrière-pays, Philippe pour sa part n'est même pas parvenu à survivre. Sans doute parce qu'il n'a pas voulu s'assujettir au Bien-être social qui, dans les trois romans, constitue un révélateur important. Qu'on en juge plutôt. $A$ en croire Sainclair (la Marche des grands cocus), "la moitié de la population est sur l'assistance sociale" (p. 58). Cette affirmation d'un fonctionnaire “indécrotablement honnête", entièrement dévoué à ses engagements professionnels et pas du tout enclin à la démagogie, équivaut évidemment à un aveu considérable: celui de l'échec de la mise en application du Plan sinon du Plan lui-même. Il est par ailleurs intéressant de voir comment le «Bien-être» départage les gens en trois catégories: outre ceux qui se résignent à recevoir une aide dont ils ne sauraient absolument pas se passer, il y en a d'autres qui par dignité personnelle la refusent. Tel est le cas, nous l'avons vu, du suicidé Philippe. C'est aussi celui de Benoît qui proclame sa fierté de ne pas "connaître la honte de vivre au crochet du gouvernement " (TM, p. 24). L'attitude de Thyme est plus ambiguë, voire nettement pharisaïque: tout en se lamentant "l'monde é trop lâche, pus parsonne veut travailler, i aiment mieux ête sul bien ête social» (VL, p. 103) - il n'hésite pas à tirer parti de la situation et se range du même coup dans la troisième catégorie, celle des gens qui profitent du système. II a en effet engagé, pour.l'entretien de son camping, deux assistés sociaux, Ti-Dré et son frère Ti-Rèle, un infirme. II les fait travailler comme des mercenaires pour un salaire dérisoire tout en se livrant au chantage: lorsqu'ils rechignent trop, il menace de les dénoncer pour leur faire perdre leurs allocations. Mais le chantage se retourne contre lui puisque Ti-Rèle menaçe à son tour Thyme de le dénoncer parce qu'il ne lui verse pas le salaire minimum!

Voilà donc dans quel contexte d'aliénation socio-économique se débattent les personnages de nos romans: "un pays où la vie s'est arrêtée", "un pays qui meurt", «un pays qui n'a pas d'avenir parce qu'il n'a pas de présent» (TM, p. 32, 23, 29); un pays où «si tous ceux qui sont sul sarvice social auraient ène cloche dans l'cou ça son'rait fort en c..." (VL, p. 49). C'est pourtant ce même «maudit pays», cette même «maudite 
terre" que les contestataires chantent en marchant sur Québec (MC, p. 140). Ne nous méprenons toutefois pas: l'amour du pays se confond ici volontiers avec la force de l'habitude comme le laisse entendre l'un des marcheurs:

Tu sumes tous les printemps dans ta mauvaise terre, quand même que tu sais que tu vas pas récolter grand-chose à l'automne. Mais tu sumes pareil! Y'a des choses qu'on fait en se farmant les yeux parce qu'y a pas d'autre chose à faire (MC, p. 194).

On voit dans quel sens l'habitude elle-même s'enracine dans un profond sentiment de fatalisme que le citadin Lucien n'a pas tardé à déceler:

Les gens du Bas-du-Fleuve [sont] en train de se faire mourir au travail pour rien... Pour rien du tout, parce que dans le ciel du Bas-duFleuve, il était écrit depuis des siècles qu'on ne devait pas tenter de s'élancer vers le monde, vers la réussite [...] Dans ce ciel, il était écrit qu'on devait retomber sans cesse et se casser les reins. (MC, p. 77).

Nés pour un petit pain, en somme. Or ce pessimisme, Isabelle ne le partage pas du tout. Elle est persuadée au contraire que «le monde est en train de changer pour de bon" (MC, p. 77) et c'est dans cette perspective que pour précipiter les choses dans le coin de pays qui est le sien, elle propose et anime la grande marche. Ce geste "révolutionnaire" n'est cependant pas uniquement motivé par l'état lamentable de la situation économique; il prend aussi origine dans une autre forme d'humiliation plus dégradante encore. Adolescente; Isabelle fut en effet violée par un prêtre. De là à faire de ce prêtre le symbole de l'aliénation religieuse il n'y avait qu'un pas, assez grand à vrai dire, que la jeune fille néanmoins franchit fort allègrement. Compte tenu de l'aspect particulièrement odieux de l'expérience qu'elle a vécue, on s'explique à la rigueur l'agressivité qu'elle met à souhaiter qu' «il ne reste plus rien de ce que les vieux ont construit à coups de goupillon, à coups de cierges, à coups de résignation " (MC, p. 57). Ce qui surprend, c'est l'ampleur et la violence de l'écho que suscitent ces propos chez la horde des marcheurs. II faut entendre en quels termes dégageant une odeur de soufre, les cultivateurs accusent l'Église d'avoir exploité leur crédulité :

Le carême, pas de viande le vendredi, la basse messe, la grand'messe puis les vêpres, et les mauvais regards et les mauvais touchers, le devoir conjugal, 12 enfants, 15 enfants, la machine à fabriquer la misère. Le devoir! Toujours le devoir! ASSEZ! (MC, p. 90).

Dans le contexte d'un état d'exprit aussi survolté et qui semble généralisé, lorsqu'lsabelle tue le prêtre indigne dans un motel de Baie-des-Mouettes, son geste prend la dimension d'un rite d'exorcisme laïque.

II n'y a pas de procès de l'institution ecclésiastique dans À travers la mer. On y perçoit plutôt l'évocation discrète mais efficacement symbolique d'un phénomène souterrain, celui de la désaffection religieuse. Par exemple, la petite église de Pabok est isolée et les terrains qui l'avoisinent ne se vendent pas. «Pourquoi, se demande Benoît, ces villageois n'avaientils jamais tenu à se presser autour du clocher? " (p. 13). Peut-être parce 
qu'ils n'étaient pas tellement différents de lui qui "se savait l'héritier d'une lignée qui ne tenait pas à s'attacher au curé... " (ibid.).

Dans C't'à cause qu'y vont su'a lune, la religion se confond avec la magie et l'eau bénite s'accommode fort bien du gin. Si Thyme se confesse et communie tous les dimanches, c'est pour neutraliser les puissances occultes qui, sait-on jamais, pourraient s'acharner contre son camping dont il fait dûment bénir l'ouverture annuellement. De son côté, le curé Bourdages, tout en s'opposant à l'implantation d'une taverne à SainteAnne-des-Monts, sait apprécier les petits coups de gin que Thyme lui 'procure de temps en temps pour le garder de son côté. Sans être à proprement parler anticlérical, Thyme se montre quelque peu scandalisé par l'aisance matérielle affichée par son curé qui non seulement loge dans un spacieux presbytère mais possède beaucoup d'argent. Thyme rêve même de pouvoir «l'acheter" un jour! En réalité, le curé Bourdages est un ecclésiastique déphasé et un peu grotesque. Sa prédication est insignifiante au point que même un illétré comme Thyme se rend compte qu'elle ne parvient pas à établir de liens convaincants entre l'Évangile et la réalité quotidienne.

Ainsi, dans les trois romans, pour des raisons et à des degrés divers, la religion n'apparaît plus - c'est le moins qu'on puisse dire - comme le référent qu'elle était par tradition. On peut dès lors se demander si dans l'univers romanesque dont il est ici question, le Plan n'était pas appelé à instaurer un nouvel ordre socio-économique destiné à prendre le relai de l'ordre religieux discrédité.

Tel semble bien être le cas si, par comparaison d'abord avec le comportement du clergé, on en juge par la sincérité et la foncière bonne volonté des fonctionnaires Lucien et surtout Sainclair. Ce dernier, originaire de la région, en connaît très bien les difficultés particulières. Mais il a beau être un directeur régional intelligent et honnête, il n'a aucun pouvoir pour apporter des solutions aux problèmes des gens. Il est forcé de passer le plus clair de son temps à rédiger des rapports et il doit sans cesse en référer aux diverses instances ministérielles des gouvernements provincial et fédéral. Devant le suicide de Philippe, il devient évident pour lui que les choses ne peuvent plus continuer ainsi. II n'est donc pas loin de partager les sentiments d'hostilité de la population rassemblée par lui à Rimouski et qui, avant de décider de marcher sur Québec conspue Larue, le directeur de l'information du Conseil général. Mais que dit au juste Larue au micro?

[II] se mit à parler du Plan, des emplois qui étaient en vue, des accords signés par les deux gouvernements, des millions de dollars à la disposition du Bas St-Laurent, des conseils régionaux, des comités locaux, etc. (MC, p. 103).

Par leurs hurlements et dans la rigolade générale, les 10000 (!) personnes rassemblées mettent fin à ce délire bureaucratique et forcent Larue à regagner son siège.

Dans le seul roman la Marche des grands cocus, il est ainsi fait référence une bonne douzaine de fois au Plan, la plupart du temps pour 
mettre en évidence le fait qu'il est “déconnecté" de la réalité, qu'il est devenu un être de raison. Encore plus souvent (plus d'une cinquantaine de fois!) c'est "le Ministère" [sic] qui est visé et dénoncé. Un tel dépit s'explique: alors qu'on avait voulu voir dans le Plan une nouvelle providence, on n'y retrouve qu'une gigantesque machine qui tourne à vide.

Dans le roman de Charles Soucy, Benoît n'est pas tendre lui non plus pour les planificateurs patentés qui ont fait miroiter aux yeux de la population les bienfaits douteux de la relocalisation. "lls chasseraient la morue, dit-il, s'ils le pouvaient ( $T M$, p. 42). De là son mépris des «fossoyeurs importés qui [viennent] défendre leur thèse sur les rochers gaspésiens " (ibid.).

Pour sa part, Thyme ne se réfère jamais explicitement au Plan, mais ne serait-ce qu'à travers ses lamentations sur l'état déplorable des routes et le Bien-être social, on sent chez lui aussi beaucoup d'amertune en face des piètres résultats obtenus par le BAEQ.

Ainsi, nous nous retrouvons dans in monde qui d'une part est en rupture avec l'ordre religieux traditionnel et qui, d'autre part, échappe à cette "vue de l'esprit " qu'est devenu le Plan au niveau socio-économique. Comme par ailleurs I'habileté politicienne tient lieu ici de politique, en l'absence de toute forme de transcendance de ces trois ordres, l'univers de nos romans qui aurait pu être tragique est en définitive un univers absurde et il en présente plusieurs symptômes.

Sur le plan du langage d'abord. S'en prendre au mode de communication le plus humain qui soit est évidemment le moyen le plus sûr de mettre en évidence le caractère absurde de l'existence. Dans nos romans, ou bien les mots manquent et alors le silence s'installe, envahissant et corrosif. C'est ce qui se produit dans la vie de Benoît et Anna. Ou bien les mots "n'ont pas de sens" comme le constate Lucien dans la Marche des grands cocus (p. 108). Pendant vingt ans, Thyme et Henrietta ont eux aussi pratiqué un langage in-signifiant fait de bouts de phrases mal rafistolées comme "belle caisse à souère... vouère... à Cap-Chat... pus d'place sul terrain..." " (VL, p. 145). Pour la première fois, ces mots sont sur le point d'avoir un sens puisque pour la première fois Thyme peut afficher complet à l'entrée de son camping. Mais il se passe alors un peu ce qui arrive dans la pièce d'lonesco, Amédée ou Comment s'en débarrasser: pour le couple qui ne communique plus, qui ne fait que coexister par la force de l'habitude, il suffit que les mots menacent de se remettre à signifier pour que le moindre événement se transforme en catastrophe... Dans le cas d'Amédée et Madeleine, il s'agit de la réception d'une lettre qui leur est bel et bien adressée. Ils ne peuvent et ne veulent pas y croire: “C'est effrayant", gémit Madeleine. On les voit donc s'obstiner à convaincre le facteur que la lettre en question ne leur est pas destinée puisque un tiers dẹs Parisiens s'appellent Amédée Buccinioni et que d'ailleurs eux ils habitent «au 29 rue des Généraux, non au 29 rue des Généraux ${ }^{13}$ "! Dans le cas de Thyme et Henrietta, le retour du langage à la signification coïncide absurdement avec le ratage de l'éclipse... 
Pas plus que les homélies du curé Bourdages, les paroles du Premier ministre aux marcheurs n'ont, elles non plus, de sens:

Vous avez devant vous le chef d'un gouvernement qui ne pense qu'à la paix et à la joie de vivre qu'elle apporte. Je suis donc ouvert, je l'ai déclaré souvent depuis le début de votre longue marche, je suis ouvert à vos suggestions, au dialogue, à la collaboration et à la participation... (MC, p. 235).

La distance qui sépare ce langage creux des préoccupations économiques et sociales de la population est telle qu'elle engendre chez ceux qui entendent ce discourś un silence de stupéfaction. Dès lors, l'absurde passe au plan des actes.

C'est en effet tout de suite après avoir entendu ces paroles que Lucien s'empare de la carabine de Josué, tire sur le ministre de l'Agriculture et le tue. Geste purement démentiel dans les circonstances puisqu'il est sans rapport avec la personne visée et uniquement motivé par le sentiment d'impuissance sexuelle de Lucien devant la virilité de Josué de qui isabelle s'est rapprochée au cours de la grande marche.

Absurde aussi l'attente de Georges et celle des Rimouskois parce qu'elle ne débouche sur rien. «Rien ne se passe, personne ne vient... C'est terrible", dit "Estragon à Gogo dans la pièce de Beckett ${ }^{14}$. Dans À travers la mer, Benoît, au hasard d'une rencontre, demande à son ami Georges: "Qu'est-ce que tu fais?" Et ce dernier, comme s'il attendait lui aussi Godot, répond: “Rien, j'attends» (p. 22). Il attend en effet qu'on agrandisse l'usine. Agrandissement promis d'année en année mais jamais réalisé. Les Rimouskois, eux, «contemplent l'immensité du fleuve en attendant une grosse industrie..." (MC, p. 25). Est-il besoin de préciser qu'ils l'attendent toujours?

II n'est pas surprenant que dans ces conditions, nos trois romans se terminent à un premier niveau, celui de l'action, sur trois échecs : échec de la révolte des marcheurs, échec de la transplantation de Benoît à Montréal, échec du projet de rentabilisation du camping de Thyme. Mais en y regardant d'un peu plus près, nous avons pu entrevoir de nous retrouver en présence d'un triple échec à un second degré, celui de la communication et de l'amour malgré les pulsions et les explosions d'érotisme. La-liaison de Lucien et d'Isabelle se dissout dans le geste insensé de Lucien tuant par dépit le ministre de l'Agriculture et se faisant tuer lui-même dans la riposte. La passade d'Isabelle et de Josué se termine à son tour par le suicide déguisé d'Isabelle. A Montréal, le couple Benoît-Anna se désagrège lui aussi à force de silence, d'habitude puis de haine. Enfin, le couple Thyme-Henrietta n'évolue guère mieux. Femme silencieuse et terre à terre, Henrietta freine constamment les élans d'optimisme de son mari. Au lit, elle lui tourne le dos. Sous ses faux airs de père Gédéon, Thyme dissimule mal son drame conjugal. 
Henrietta n'en finissait plus de se renfermer dans un silence devenu lourd à supporter à la longue. Pendant ces moments, il lui prenait de secrètes envies de coucher sa femme sur le plancher et de la frapper jusqu'à ce qu'elle se mette à parler sans arrêt. Mais Thyme n'avait jamais osé coucher sa femme sur le plancher, car peut-être alors en aurait-elle trop dit, des paroles qu'il lui aurait fait mal d'entendre et pour lesquelles il aurait eu du remords. II n'avait jamais osé (VL, p. 104).

Le drame ne s'extériorise cependant pas et, à la fin du roman, on retrouve Henrietta en train de lire son journal assise au bout de la table, selon son habitude; et Thyme boit un grand verre d'eau, comme d'habitude, avant d'aller se coucher...

Dans les trois romans, l'incommunicabilité entre les êtres est liée à une forme ou l'autre de stérilité ou d'impuissance. Par exemple, c'est l'impuissance sexuelle de Lucien, signe et aboutissement de son manque de virilité sur le plan «révolutionnaire» qui est à l'origine du refroidissement progressif d'Isabelle à son égard au profit ultime et éphémère, il est vrai, de cette force de la nature qu'est Josué; il était donc naturel de voir ce couple hautement érotique prendre l'initiative de la révolte. Dans $\grave{A}$ travers la mer, le couple est sans enfants. Benoît croit que sa femme est stérile jusqu'au jour où un médecin lui apprend qu'au contraire, c'est lui qui l'est. D'une manière très significative, c'est seulement après l'avoir appris que Benoît capitule devant Anna à qui revient alors la discutable initiative du déménagement à Montréal, projet auquel son mari s'était jusque-là farouchement opposé. Sans enfants également le couple ThymeHenrietta; mais cette fois, du fait d'Henrietta. Or nous avons déjà observé le rôle d'éteignioir joué par cette dernière auprès d'un Thyme volontiers idéaliste qui essaie de s'en sortir en visant haut: attirer les touristes en Gaspésie «à longueur d'année» (VL, p. 126). Ni plus ni moins!

S'il est vrai que "la limite de l'imaginable serait un monde complètement humanisé ${ }^{15}$ ", il va sans dire que nous sommes ici loin du compte! En s'inspirant d'une collectivité au contraire fortement déshumanisée, nos romanciers ont créé un univers dont la circularité presque schizophrénique est polarisée par l'échec d'une planification socio-économique qui avait pourtant au départ fait naître beaucoup d'enthousiasme et mobilisé bien des énergies. La mythologie littéraire qui émerge ainsi de l'image en creux des conditions de vie de toute une région pour devenir le symbole du désespoir et de la désintégration ne doit cependant pas être interprétée comme le signe d'une fin mais plutôt comme le commencement d'une prise de conscience que le narrateur de la Marche des grands cocus évoque dans les termes suivants empruntés à Jung:

Tout devient relatif et par conséquent douteux et tandis que la conscience, hésitante et incertaine, considère ce monde précaire, où retentissent les traités de paix et d'amitié, la démocratie et la dictature, le capitalisme et le bolchévisme, l'aspiration de l'âme s'élève et cherche une réponse dans le tumulte du doute et de l'insécurité. Et ce sont précisément les couches obscures du peuple, les silencieux dont on a tant souri, ceux qui ont été moins blessés que les sommi- 
tés brillantes de la population par les préjugés académiques, qui se laissent aller à la poussée inconsciente de l'âme... (p. 112).

\author{
Gilles Lamontagne \\ Université du Québec à Rimouski
}

1. Montréal, L'Actuelle, 1972, 265 p. Pour les références à ce roman, l'abréviation MC sera utilisée.

2. Montréal, L'Actuelle, 1974, 163 p. Abréviation: VL.

3. Montréal, Le Cercle du Livre de France, 1974, 144 p. Abréviation: TM.

4. Dans un ouvrage intitulé l'Est du Québec à l'heure du développement régional (Rimouski, Cahiers de I'UQAR, $n^{\circ} 1,1973,319$ p.) mon collegue Clermont Dugas tente d'évaluer la mise en application du Plan dix ans après la création du BAEQ. Avec l'aimable autorisation de l'auteur, que je remercie cordialement, j'ai puisé dans cette étude les éléments d'information relative au Plan dont j'avais besoin pour mon essai.

5. C. Dugas, le Dovveloppement..., p. 170.

6. L'imaginaire, c'est bien connu, précède la réalité. N'a-t-on pas vu il y a quelques années; les agriculteurs de la région de Rimouski immoler plusieurs têtes de bétail sur la place du Centre commercial Cooprix en guise de protestation contre les politiques agricoles des gouvernements?

7. Ici encore l'imagination du romancier est en avance sur les événements. En effet, au cours de l'assemblée générale annuelle de l'Opération Dignité tenue à Amqui le 1er mai 1976, au moment où les participants envisageaient différents moyens d'action pour faire bouger les gouvernements, «le député De Bané y est allé de sa suggestion: une marche d'une dizaine de représentants de paroisses, marche qui les conduirait à Québec et Ottawa et qui devrait durer tant et aussi longtemps que les autorités gouvernementales n'auraient pas donné de réponse satisfaisante " (extrait d'un reportage de Damien Gagnon, le Soleil, 3 mai 1976, section C, p. 2). S'il se montre timide quant au nombre de «marcheurs " qu'il propose, on voit que le député n'est pas en reste par rapport au romancier au sujet de la distance à parcourir.

8. C. Dugas, le Développement..., p. 213.

9. Ibid., p. 226.

10. Ibid., p. 195.

11. M. Dugas note à ce sujet: “Nous n'avons pas pu obtenir de l'administration gouvernementale les informations requises pour faire une analyse complète de ce secteur. Les éléments qui sont présentés dans le présent travail ne visent qu'à situer de façon très générale la pêche parmi les autres secteurs d'activité" (p. 193).

12. C. Dugas, le Développement..., p. 197.

13. Eugène lonesco, Théatre, Paris, Gallimard, 1963, t. 1, p. $259-260$.

14. Samuel Beckett, En attendant Godot, Paris, Éd. de Minuit, 1970, p. 70.

15. Northrop Frye, Pouvoirs de l'imagination, Montréal, HMH, 1969, p. 33. 